PROCEEDINGS OF THE

AMERICAN MATHEMATICAL SOCIETY

Volume 127, Number 1, January 1999, Pages 63-69

S 0002-9939(99)04530-X

\title{
$G$-IDENTITIES ON ASSOCIATIVE ALGEBRAS
}

\author{
Y. BAHTURIN, A. GIAMBRUNO, AND M. ZAICEV \\ (Communicated by Ken Goodearl)
}

\begin{abstract}
Let $R$ be an algebra over a field and $G$ a finite group of automorphisms and anti-automorphisms of $R$. We prove that if $R$ satisfies an essential $G$-polynomial identity of degree $d$, then the $G$-codimensions of $R$ are exponentially bounded and $R$ satisfies a polynomial identity whose degree is bounded by an explicit function of $d$. As a consequence we show that if $R$ is an algebra with involution $*$ satisfying a $*$-polynomial identity of degree $d$, then the *-codimensions of $R$ are exponentially bounded; this gives a new proof of a theorem of Amitsur stating that in this case $R$ must satisfy a polynomial identity and we can now give an upper bound on the degree of this identity.
\end{abstract}

\section{$\S 1$. INTRODUCTION}

Let $R$ be an algebra over a field $F$ and $G$ a finite group of automorphisms and anti-automorphisms of $R$. $G$-polynomials and $G$-polynomial identities are defined in a natural way (see $[\mathrm{M}]$ and $[\mathrm{GR}]$ ). Two kinds of problems are usually considered:

(1) Suppose that the ring of invariants $R^{G}$ satisfies a polynomial identity or more generally that $R$ satisfies a $G$-polynomial identity of degree $d$; under what circumstances must $R$ also satisfy a polynomial identity ?

(2) In the case of hypotheses giving a positive solution to the above problem, can one find an upper bound for the degree of a polynomial identity of $R$ (as a function of $d$ )?

It is well known (see $[\mathrm{M}]$ ) that problem 1 has a negative answer in general. In case $G$ is a group of automorphisms and $R$ has no $|G|$-torsion, Kharchenko [K] proved that a polynomial identity (PI) in $R^{G}$ forces the existence of a polynomial identity in $R$. Let $*$ denote an involution (an anti-automorphism of order 2) on $R$; when $G=\{1, *\}$, Amitsur in two subsequent papers ([A1] and [A2]) gave a positive answer to problem 1 with no further hypotheses on $R$.

We should remark that, if $G$ is arbitrary, by combining the results of Amitsur and Kharchenko it is easy to prove that $R^{G}$ PI forces $R$ PI in case of no $|G|$-torsion.

What about problem 2 ? Very little is known for general algebras. Both Amitsur and Kharchenko gave bounds on the degree of a polynomial identity satisfied by $R$ provided $R$ is a semiprime algebra. While passing from semiprime algebras to arbitrary ones (by using the famous Amitsur's trick) it was proved that $R$ must satisfy an identity of the form $S_{|G| d}\left(x_{1}, \ldots, x_{|G| d}\right)^{m}$ where $S_{|G| d}\left(x_{1}, \ldots, x_{|G| d}\right)$ is the

Received by the editors December 18, 1996 and, in revised form, May 13, 1997.

1991 Mathematics Subject Classification. Primary 16R50; Secondary 16W20.

Y. Bahturin and M. Zaicev acknowledge support by the Russian Foundation of Fundamental Research, grant 96-01-00146. A. Giambruno was supported by MURST and CNR of Italy. 
standard polynomial of degree $|G| d$. Thus no information on $m$ was available and, so, no dependence between $d$ and the degree of an identity on $R$ was established.

In this paper we will approach problem 1 (and 2) by translating it into a problem concerning the $(G$-)codimensions of the algebra $R$. The sequence of codimensions was introduced by Regev in [Re1] as a basic tool for proving the tensor product theorem and it was applied in [Re2] for finding explicit identities of a PI-algebra. A basic theorem proved by Regev states that an algebra $R$ satisfies a PI if and only if the codimensions of $R$ are exponentially bounded. The sequence of $G$-codimension was introduced and studied in [GR].

For an algebra $R$ let $c_{n}(R)$ and $c_{n}(R \mid G)$ denote the $n$-th codimension and the $n$-th $G$-codimension respectively of $R$. In this paper we introduce the notion of an essential $G$-polynomial and we prove that if $R$ satisfies an essential $G$-identity of degree $d$, then $c_{n}(R \mid G)$ and, so, $c_{n}(R)$ is bounded by the exponential function $|G|^{n}(f(d,|G|)-1)^{2 n}$ where $f(d,|G|)$ is explicitly computed. It also follows that $f(d,|G|)$ is an upper bound for the degree of a polynomial identity satisfied by $R$.

As a consequence we give a positive solution to a question raised in [GR], namely we prove that if $R$ is an algebra with involution $*$ satisfying a $*$-polynomial identity of degree $d$, the *-codimensions $c_{n}(R \mid *)$ of $R$ are exponentially bounded by a function of $d$. This gives a new proof of Amitsur's theorems and we can now give an upper bound on the degree of a PI satisfied by $R$.

In order to bound the codimensions, we use and extend a result of Latyshev; our main tools are the properties of $m$-indecomposable words introduced and studied by Razmyslov (see $[R]$ ) and we are now able to find an estimate on their number (namely Lemma 2).

One final remark is in order. As a consequence of this estimate the results of [BZ] are improved in that there is now an explicit formula that gives an upper bound for the degree of the identity satisfied by a Lie (super)algebra $L$ graded by a finite group $G$ of order $t$ if the trivial component of $L$ satisfies a non-trivial identity of degree $d$ and this bound depends on $t$ and $d$ entirely.

\section{§2. $G$-Polynomials And $G$-IDEntities}

Throughout $\operatorname{Aut}^{*}(R)$ will be the group of automorphisms and anti-automorphisms of the $F$-algebra $R$ and $G \leq \operatorname{Aut}^{*}(R)$ a finite group. If $\operatorname{Aut}(R)$ is the group of automorphisms of $R$, then $G \cap \operatorname{Aut}(R)$ is a subgroup of $G$ of index $\leq 2$.

Let $X$ be a set, $G$ a finite group and $H$ a subgroup of $G$ of index two. If we interpret $H$ as automorphisms and $G \backslash H$ as anti-automorphisms, we can construct $F\langle X \mid G\rangle$, the free algebra on $X$ with $G$-action. $F\langle X \mid G\rangle$ is freely generated by the set $\left\{x^{g}=g(x) \mid x \in X, g \in G\right\}$ on which $G$ acts in a natural way: $\left(x^{g_{1}}\right)^{g_{2}}=x^{\left(g_{2} g_{1}\right)}$. Extend this action to $F\langle X \mid G\rangle$ : if $v$ and $w$ are monomials, $g \in G$, then $(v w)^{g}=$ $v^{g} w^{g}$ if $g \in H$ and $(v w)^{g}=w^{g} v^{g}$ if $g \in G \backslash H$. By linearity now $G$ acts on $F\langle X \mid G\rangle$ with $H$ as automorphisms and $G \backslash H$ as anti-automorphisms. Given any algebra $R$ as above, by interpreting $G \leq \operatorname{Aut}^{*}(R)$ and $H=G \cap \operatorname{Aut}(R)$, any set theoretic map $\phi: X \mapsto R$ extends uniquely to a homomorphism $\bar{\phi}: F\langle X \mid G\rangle \mapsto R$ such that $\bar{\phi}\left(x^{g}\right)=\phi(x)^{g}$. For fixed $R$, let $\bar{\Phi}$ be the set of all such homomorphisms and set

$$
I=\bigcap_{\bar{\phi} \in \bar{\Phi}} \operatorname{Ker} \bar{\phi} .
$$


An element $f \in F\langle X \mid G\rangle$ will be called a $G$-polynomial. If $f \in I$, then $f$ will be called a $G$-identity for $R$.

Let $G^{n}=G \times \cdots \times G$ and $g=\left(g_{1}, \ldots, g_{n}\right) \in G^{n}$. Denote by

$$
P_{n, g}=\operatorname{Span}_{F}\left\{x_{\sigma(1)}^{g_{\sigma(1)}} \cdots x_{\sigma(n)}^{g_{\sigma(n)}} \mid \sigma \in S_{n}\right\}
$$

the space of multilinear polynomials in $F\langle X \mid G\rangle$ in the variables $x_{1}^{g_{1}}, \ldots, x_{n}^{g_{n}}$. In particular, for $1=(1, \ldots, 1)$ we have $P_{n, 1}=P_{n}$. Also let $Q_{n}=\sum_{g \in G^{n}} P_{n, g}$ be the space of multilinear $G$-polynomials in $x_{1}, \ldots, x_{n}$.

A $G$-identity $f \in Q_{n}$ will be called an essential one if it is of the form

$$
f=x_{1}^{1} \cdots x_{n}^{1}+\sum_{\substack{1 \neq \sigma \in S_{n} \\ g \in G^{n}}} \alpha_{\sigma, g} x_{\sigma(1)}^{g_{1}} \cdots x_{\sigma(n)}^{g_{n}} .
$$

If we let $J \subset F\langle X\rangle \subset F\langle X \mid G\rangle$ be the T-ideal of identities of $R$, then $c_{n}(R)=$ $\operatorname{dim} \frac{P n+J}{J}$ and $c_{n}(R \mid G)=\operatorname{dim} \frac{Q_{n}+I}{I}$ are called the $n$-th codimension of $R$ and the $n$th $G$-codimension of $R$, respectively. The relation between these two dimensions is given in the following ([GR, Lemma 4.4])

Lemma 1. $c_{n}(R) \leq c_{n}(R \mid G)$.

\section{$\S 3$. Decomposable monomials}

Introduce a partial ordering on the variables $x_{i}^{g}$ by requiring that $x_{i}^{g}<x_{j}^{h}$ if $i<j$ for $g, h \in G$; extend this ordering lexicographically to all monomials (words) in $Q_{n}$ by comparing them from left to right.

Following $[\mathrm{R}]$ we introduce the following:

Definition. A monomial $w \in P_{n, g}$ is said to be $m$-decomposable if it can be represented in the form $w=a w_{m} w_{m-1} \cdots w_{1} b$ where $w_{i}(i=1, \ldots, m)$ are nonempty monomials such that

a) the left variable in the monomial $w_{i}$ is greater than any other variable in this monomial $(i=1, \ldots, m)$;

b) the first variable in the monomial $w_{i+1}$ is greater than the first variable in the monomial $w_{i}(i=1, \ldots, m-1)$.

In case $w$ has no $m$-decompositions then it is said to be $m$-indecomposable.

In this section we want to find a bound on the number $a_{m}(n)$ of $m$-indecomposable multilinear monomials in $x_{1}, \ldots, x_{n}$. It is well known that $a_{m}(n)$ satisfies the following recurrent relation (see [R, Section 2.1])

$$
a_{m}(n)=\sum_{i=0}^{n-1} \frac{(n-1) !}{i !(n-1-i) !} a_{m}(i) a_{m-1}(n-1-i),
$$

where $a_{m}(0)=1$. Moreover if we set

$$
b_{m}(n)=\frac{a_{m}(n)}{n !}
$$

asymptotically the value $b_{m}(n)$ is less than $\left(\frac{1}{c}\right)^{n}$ for any fixed $c$. We will need an explicit estimate for $n$, depending only on $c$ and $m$.

Let $t=m+\left[\log _{2} c\right]$ and $N=2^{t 2^{t+1}}$. We denote by $p_{j}, j \geq 3$ an integer for which

$$
\underbrace{\log _{N} \ldots \log _{N}}_{j-2} p_{j}=p_{2}
$$


and set $p_{2}=2^{t 2^{t}}$. We remark that with such a choice of $p_{2}$ the inequality

$$
n ! p_{2}>2^{t n}
$$

is satisfied for all natural $n$. We set $f(m, c)=\log _{2} p_{m}$.

Lemma 2. Let $n \geq f(m, c)$. Then $b_{m}(n)<\left(\frac{1}{c}\right)^{n}$.

Proof. From (1) above it follows that $b_{k}(n)$ satisfies the following recurrent relation

$$
b_{k}(n)=\frac{1}{n} \sum_{i=0}^{n-1} b_{k}(i) b_{k-1}(n-1-i),
$$

where $b_{k}(0)=1$. It is easy to observe, that $b_{2}(n)=\frac{1}{n !}$. In what follows we assume that $k \geq 2$.

First, we show that if the following conditions are satisfied

$$
\begin{gathered}
b_{k}(j)<p \varepsilon^{j}, j=0,1, \ldots \\
b_{k+1}(j)<q(2 \varepsilon)^{j}, j=0,1, \ldots, n-1,
\end{gathered}
$$

where $\varepsilon<\frac{1}{2}$, then

$$
b_{k+1}(n)<\frac{2 p q}{n}(2 \varepsilon)^{n-1} .
$$

In fact, it follows from (3) that

$$
b_{k+1}(n)<\frac{1}{n} \sum_{i=0}^{n-1} q(2 \varepsilon)^{i} p \varepsilon^{n-1-i}=\frac{p q}{n}(2 \varepsilon)^{n-1} \sum_{i=0}^{n-1}\left(\frac{1}{2}\right)^{i}<\frac{2 p q}{n}(2 \varepsilon)^{n-1} .
$$

We assume now, that (4) holds, $n_{0}>\frac{p}{\varepsilon}$ and $q \geq\left(\frac{1}{2 \varepsilon}\right)^{n_{0}}>\left(\frac{1}{2 \varepsilon}\right)^{\frac{p}{\varepsilon}}$. Then the inequality (5) is satisfied for all $j \leq n_{0}$, since $b_{k}(n) \leq 1$ for all $k$ and $n$. But then, by $(6), b_{k+1}(j)<q(2 \varepsilon)^{j}$ also for $j=n_{0}+1$, since $\frac{2 p}{n_{0}} \leq 2 \varepsilon$. It follows that with such a choice of $q$ the inequality (5) holds for all $j$. So, we have shown that if

$$
b_{k}(j)<q_{k} \varepsilon_{k}^{j}
$$

for all $j$, then

$$
b_{k+1}(j)<q_{k+1} \varepsilon_{k+1}^{j}
$$

also for all $j$ with $\varepsilon_{k+1}=2 \varepsilon_{k}$ and

$$
q_{k+1} \geq\left(\frac{1}{\varepsilon_{k+1}}\right)^{\frac{q_{k}}{\varepsilon_{k}}}
$$

We set $\varepsilon_{2}=2^{-t}$. From (2) it follows that $b_{2}(j)<p_{2} \varepsilon_{2}^{j}$ for all $j$. Then $\varepsilon_{k+1}=2^{-r}$ and $r \leq t$. Hence

$$
\left(\frac{1}{\varepsilon_{k+1}}\right)^{\frac{q_{k}}{\varepsilon_{k}}}=\left(2^{r 2^{r+1}}\right)^{q_{k}} \leq N^{q_{k}} .
$$

Thus the inequality (7) is satisfied by all numbers of the form $q_{2}=p_{2}, \ldots, q_{k}=$ $p_{k}, q_{k+1}=N^{p_{k}}=p_{k+1}$. Hence, for $k=m$ we have

$$
b_{m}(n)<p_{m} \varepsilon_{m}^{n} \quad \text { and } \quad \varepsilon_{m}=2^{m-2} \varepsilon_{2}=2^{m-t-2} .
$$


By the choice of the number $t$ the following inequality holds:

$$
t \geq m+\log _{2} c-1=m+\log _{2} 2 c-2 .
$$

Hence $\varepsilon_{m} \leq \frac{1}{2 c}$. But then

$$
b_{m}(n)<p_{m}\left(\frac{1}{2 c}\right)^{n} \text {. }
$$

By the hypothesis, $n \geq \log _{2} p_{m}$. Hence $p_{m}\left(\frac{1}{2}\right)^{n} \leq 1$ and from (8) we obtain the required estimate. Lemma 2 has been proven.

\section{$\S 4$. MAIN RESUlT}

In this section we shall prove the result on $G$-identities mentioned in the introduction and then we shall deduce Amitsur's theorem [A1] on rings with involution with the desired bound on the degree of a PI for $R$.

Theorem 1. Let $R$ be an algebra over a field $F$ and $G$ a finite subgroup of Aut* $(R)$. Suppose that $R$ satisfies some mutilinear essential $G$-identity of degree $d$. Then for $n$ sufficiently large we have $c_{n}(R \mid G) \leq|G|^{n}(f(d,|G|)-1)^{2 n}$ and $R$ satisfies a nontrivial polynomial identity, whose degree is bounded by the function $f(d,|G|)$. In case $G \leq \operatorname{Aut}(R)$, then $c_{n}(R \mid G) \leq|G|^{n}(d-1)^{2 n}$ and $R$ satisfies a non-trivial polynomial identity, whose degree is bounded by $3|G|(d-1)^{2}$.

The proof of this theorem will be deduced after a sequence of reductions. Suppose throughout that $G$ is a finite subgroup of $\operatorname{Aut}^{*}(R)$ and $R$ satisfies an essential $G$ identity $f$ of degree $d$.

Notice that in order to prove that $R$ satisfies an ordinary identity whose degree is bounded by a function $k=k(d,|G|)$ it is enough to find an integer $n$ for which the following inequality is satisfied:

$$
\operatorname{dim} \frac{P_{n}+I}{I}<n !
$$

and to show that $n \leq k(d,|G|)$. Note that $P_{n} \subset Q_{n}$ therefore it is enough to show that

$$
c_{n}(R \mid G)=\operatorname{dim} \frac{Q_{n}+I}{I}<n ! .
$$

Let us denote by $V^{(d)}$ the linear span of $d$-indecomposable monomials.

Lemma 3. If $R$ satisfies a multilinear essential $G$-identity of degree $d$, then for any $n$ we have $Q_{n} \subset I+V^{(d)}$.

Proof. Suppose by contradiction that the Lemma is false. Then there exists a counterexample $B=x_{i_{1}}^{s_{1}} \cdots x_{i_{n}}^{s_{n}}$ which is minimal in the left lexicographic order defined in the previous section.

By the hypotheses $R$ satisfies an identity of degree $d$, hence

$$
x_{1}^{1} \cdots x_{d}^{1} \equiv \sum_{\substack{1 \neq \sigma \in S_{d} \\ g \in G^{d}}} \alpha_{\sigma, g} x_{\sigma(1)}^{g_{1}} \cdots x_{\sigma(d)}^{g_{d}} \quad(\bmod I),
$$

for some $\alpha_{\sigma, g} \in F$. 
Since the ideal of $G$-identities of $R$ is invariant under all endomorphisms of $F\langle X \mid G\rangle$ commuting with the $G$-action (i.e., $\left.\theta\left(a^{g}\right)=\theta(a)^{g}\right)$, (11) implies that

$$
t_{1} \cdots t_{d} \equiv \sum_{\substack{1 \neq \sigma \in S_{d} \\ g \in G^{d}}} \alpha_{\sigma, g} t_{\sigma(1)}^{g_{1}} \cdots t_{\sigma(d)}^{g_{d}}(\bmod I)
$$

for any $t_{1}, \ldots, t_{n} \in F\langle X \mid G\rangle$.

Since $B \notin V^{(d)}$, there exist indices $j_{1}, \ldots, j_{d}$ which determine a $d$-decomposition on $B$. We will write $y_{1}=x_{i_{1}}^{s_{1}}, \ldots, y_{n}=x_{i_{n}}^{s_{n}}$ for convenience.

We denote by $a_{0}$ the product $y_{1} \cdots y_{j_{1}-1}$, if $j_{1}>1$. If $j_{1}=1$, then we simply assume that $a_{0}$ is the empty word. Similarly, we set $a_{d+1}=y_{j_{d}+1} \cdots y_{n}$ if $j_{d}<n$ and set $a_{d+1}$ the empty word as soon as $j_{d}=n$. For all $k=1, \ldots, d-1$ we set

$$
a_{k}=y_{j_{k}} y_{j_{k}+1} \cdots x_{j_{k+1}-1}
$$

and $a_{d}=y_{j_{d}}$. Then $B=a_{0} a_{1} \cdots a_{d} a_{d+1}$. But it follows from (12) that, modulo $I$, we can express $B$ as a linear combination of products of the form $B_{\sigma, g}=$ $a_{0} a_{\sigma(1)}^{g_{1}} \cdots a_{\sigma(d)}^{g_{d}} a_{d+1}$ where $\sigma \in S_{d}$ and $\sigma \neq 1$. Since $B$ is a minimal counterexample and all $B_{\sigma, g}$ are less than $B$, we obtain (modulo $I$ ) an expression of $B$ as a linear combination of $d$-indecomposable monomials, a contradiction.

To complete the proof of Theorem 1, notice that (modulo $I$ ) $Q_{n}$ is a sum of $|G|^{n}$ subspaces $P_{n, g}$ and every $P_{n, g}$ contains no more than $a_{d}(n)$ linearly independent monomials. By Lemma 2 it follows that the inequalities (9) and (10) hold for $n \geq f(d,|G|)$ and, so, $R$ satisfies a PI of degree $f(d,|G|)$. But then by [GR, Lemma $4.7], c_{n}(R \mid G) \leq|G|^{n} c_{n}(R) \leq|G|^{n}(f(d,|G|)-1)^{2 n}$.

In case $G \leq \operatorname{Aut}(R)$ one can give an estimate of the degree of an identity on $R$ which is better than the one given by the function $f(d,|G|)$ above. To accomplish this, one should observe that the space $Q_{n}$ can be spanned (modulo $I$ ) by the $d$ good monomials in the sense of $[\operatorname{Re} 2]$. In this case since by $[\operatorname{Re} 2$, Theorem 1.8] the number of $d$-good monomials is $\leq \frac{(d-1)^{2 n}}{(d-1) !}$ it follows that $c_{n}(R \mid G) \leq|G|^{n}(d-1)^{2 n}$ and, as in [BGR] $R$ satisfies a PI of degree $\leq e|G|(d-1)^{2}$ where $e$ is the basis of the natural logarithms.

We can now improve Amitsur's theorem. In case $G=\{1, *\}$ where $*$ is an involution, $G$-polynomials and $G$-identities are called $*$-polynomials and $*$-identities respectively. Also, $c_{n}(R \mid G)=c_{n}(R \mid *)$ is called the $n$-th $*$-codimension of $R$.

Corollary 1. Let $R$ be an algebra with involution * over a field $F$ satisfying a non-trivial *-identity of degree $d$. Then for $n$ sufficiently large we have $c_{n}(R \mid *) \leq$ $2^{n}(f(2 d, 2)-1)^{2 n}$ and $R$ satisfies a non-trivial polynomial identity whose degree is bounded by the function $f(2 d, 2)$.

Proof. By applying the usual linearization process to the $*$-identity of $R$, we get that $R$ satisfies a $*$-identity of the form

$$
\sum_{s \in G^{d}} \alpha_{s} x_{1}^{s_{1}} \cdots x_{d}^{s_{d}}+\sum_{\substack{1 \neq \sigma \in S_{d} \\ q \in G^{d}}} \beta_{\sigma, q} x_{\sigma(1)}^{q_{1}} \cdots x_{\sigma(d)}^{q_{d}}
$$

where $s=\left(s_{1}, \ldots, s_{d}\right), q=\left(q_{1}, \ldots, q_{d}\right) \in G^{d}$ and $G=\{1, *\}$. Also, without loss of generality we may assume that $\alpha_{1} \neq 0$ for $1=(1, \ldots, 1)$. Replacing $x_{i}$ by $x_{2 i-1} x_{2 i}$ 
for all $i=1, \ldots d$, we obtain a $*$-identity of the form

$$
x_{1} \cdots x_{2 d}+\sum_{\substack{1 \neq \sigma \in S_{2 d} \\ q \in G^{2 d}}} \gamma_{\sigma, q} x_{\sigma(1)}^{q_{1}} \cdots x_{\sigma(2 d)}^{q_{2 d}} .
$$

Since this is an essential $G$-identity on $R$, Theorem 1 gives the desired conclusion.

\section{REFERENCES}

[A1] S. A. Amitsur, Rings with involution, Israel J. Math. 6 (1968), 99 - 106. MR 39:256

[A2] S. A. Amitsur, Identities in rings with involution, Israel J. Math. 7 (1969), 63 - 68. MR 39:4216

[BGR] Y. Bahturin, A. Giambruno and D. Riley, Group-graded algebras with polynomial identity, Israel J. Math. 104 (1998), 145-156.

[BZ] Y. Bahturin and M. Zaicev, Identities of graded algebras, J. Algebra, to appear.

[GR] A. Giambruno and A. Regev, Wreath products and P.I. algebras, J. Pure Applied Algebra 35 (1985), 133 -149. MR 86e: 16027

[K] V. K. Kharchenko, Galois extensions and quotient rings, Algebra i Logika 13 (1974), 460 - 484 (Russian); English transl. (1975), 264 - 281. MR 53:498

[L] V. N. Latyshev, On the theorem of Regev about identities in the tensor product of P.I. algebras, Uspekhi Mat. Nauk. 27 (1972), 213 - 214 (Russian). MR 52:13924

[M] S. Montgomery, Fixed rings of finite automorphism groups of associative rings, LNM $\mathrm{n}$. 818 Springer-Verlag, Berlin, 1980. MR 81j:16041

[R] Yu. P. Razmyslov, Identities of Algebras and Their Representations, Transl. Math. Monogr., vol. 138, Amer. Math. Soc., Providence RI, 1994 xiii+318pp. MR 95i:16022

[Re1] A. Regev, Existence of identities in $A \otimes B$, Israel J. Math. 11 (1972), 131 - 152. MR 47:3442

[Re2] A. Regev, The representations of $S_{n}$ and explicit identities for P.I. algebras, J. Algebra 51 (1978), $25-40$. MR 57:9745

(Y. Bahturin and M. Zaicev) Department of Algebra, Faculty of Mathematics and MeChanics, Moscow State University, Moscow, 119899 Russia

E-mail address: bahturin@mech.math.msu.su

E-mail address: zaicev@nw.math.msu.su

(A. Giambruno) Dipartimento di Matematica e Applicazioni, Università di Palermo, Via Archirafi 34, 90123 Palermo, Italy

E-mail address: a.giambruno@unipa.it 